\title{
Critical thresholds for intracranial pressure vary over time in non-craniectomised traumatic brain injury patients
}

\author{
Basil Nourallah ${ }^{1}$. Frederick A. Zeiler ${ }^{1,2,3} \cdot$ Leanne Calviello $^{4} \cdot$ Peter Smielewski $^{4}$. \\ Marek Czosnyka $^{4,5}$. David K. Menon ${ }^{1}$
}

Received: 5 March 2018 / Accepted: 18 April 2018 / Published online: 7 May 2018

(C) The Author(s) 2018

\begin{abstract}
Background Intracranial pressure (ICP)- and cerebral perfusion pressure (CPP)-guided therapy is central to neurocritical care for traumatic brain injury (TBI) patients. We sought to identify time-dependent critical thresholds for mortality and unfavourable outcome for ICP and CPP in non-craniectomised TBI patients.

Methods This is a retrospective cohort study of 355 patients with moderate-to-severe TBI who received ICP monitoring and were managed without decompressive craniectomy in a tertiary hospital neurocritical care unit. Patients were grouped in $2 \times 2$ tables according to survival/death or favourable/unfavourable outcomes at 6 months and serial thresholds of mean ICP and CPP, using increments of 0.1 and $0.5 \mathrm{mmHg}$ respectively. Sequential chi-square analysis was performed, and the thresholds yielding the highest chi-square test statistic were taken as having the best discriminative value for outcome. This process was repeated over monitoring periods of 1, 3, 5 and 7 days and for each day of recording to establish time-dependent thresholds. The same analysis was performed for age and sex subgroups.

Results Global ICP thresholds were 21.3 and $20.5 \mathrm{mmHg}$ for mortality and unfavourable outcome respectively $(p<0.001)$. After the first day of ICP monitoring, ICP thresholds fell to between 15 and $20 \mathrm{mmHg}$ and remained significant $(p<0.05)$. Significant time-dependent CPP thresholds for mortality or unfavourable outcome were often not identified, and no identifiable trends were produced.

Conclusion Critical ICP thresholds in non-craniectomised TBI patients vary with time and fall below established ICP targets after the first day of monitoring.
\end{abstract}

Keywords Neuromonitoring $\cdot$ Neurocritical care $\cdot$ Traumatic brain injury $\cdot$ Threshold $\cdot$ Intracranial pressure $\cdot$ Cerebral perfusion pressure

Electronic supplementary material The online version of this article (https://doi.org/10.1007/s00701-018-3555-3) contains supplementary material, which is available to authorized users.

Basil Nourallah

basilnourallah@gmail.com

Frederick A. Zeiler

umzeiler@myumanitoba.ca

Leanne Calviello

lac76@cam.ac.uk

Peter Smielewski

ps10011@cam.ac.uk

Marek Czosnyka

mc141@medschl.cam.ac.uk

David K. Menon

dkm13@cam.ac.uk
1 Division of Anaesthesia, Addenbrooke's Hospital, University of Cambridge, Cambridge, UK

2 Department of Surgery, Rady Faculty of Health Sciences, University of Manitoba, Winnipeg, Canada

3 Clinician Investigator Program, Rady Faculty of Health Sciences, University of Manitoba, Winnipeg, Canada

4 Section of Brain Physics, Division of Neurosurgery, Addenbrooke's Hospital, University of Cambridge, Cambridge, UK

5 Institute of Electronic Systems, Warsaw University of Technology, Warsaw, Poland 


\section{Introduction}

Intracranial pressure (ICP) monitoring is a fundamental strategy in neurocritical care for traumatic brain injury (TBI) and is used routinely to guide medical and surgical intervention. Though well-supported by retrospective $[1,11,12]$ and prospective studies [24], there is no level I evidence for outcome benefit from treatment guided by ICP monitoring. The only randomised controlled trial showed no improvement in outcome associated with maintaining monitored ICP below $20 \mathrm{mmHg}$ compared to treatment guided by clinical and radiological assessment. Among many worthwhile caveats, the authors note that the lack of demonstrable efficacy may be attributed in part to the use of a generic, universal target ICP [9] and poor monitoring method (instant endhour values).

Intracranial hypertension is commonly defined at a threshold of 20-25 $\mathrm{mmHg}$ in clinical practice, and unfavourable outcomes have been described at ICP thresholds ranging from 15 to $25 \mathrm{mmHg}[5,10,16,20]$. Recent consensus guidelines from the Brain Trauma Foundation (BTF) specify a critical ICP threshold of $22 \mathrm{mmHg}$ below which ICP should be maintained [8], based on evidence that ICP above this value best predicts mortality and severe morbidity [22].

However, it has been suggested that optimal ICP thresholds may vary with demographic characteristics such as age and sex [22]. It also stands to reason that patients treated medically may have different ICP thresholds than those treated with decompressive craniectomy (DC), as these groups experience distinct intracranial biomechanical parameters. Other physiological variables, for example, state of autoregulation, probably play additional roles [14]. Thus, applying a universal threshold to ICP management fails to account for the heterogeneity in patient-specific injury patterns and response to treatment interventions. Of particular importance, the $22-\mathrm{mHg}$ threshold is derived from a mixed cohort of patients managed with and without DC. Surprisingly low timedependent ICP thresholds for outcome have recently been described in DC patients [19], but time-dependent ICP threshold analysis has not been conducted in an exclusive cohort of patients managed without DC (non-DC).

Similarly, BTF guidelines recommend targeting cerebral perfusion pressure (CPP) between 60 and $70 \mathrm{mmHg}$ based on available evidence [2, 8, 22], but note the growing consensus that individualised cerebral perfusion pressure thresholds aimed at achieving optimal cerebrovascular reactivity may be associated with superior outcomes $[3,23]$. The same may be true of "critical threshold' of ICP [14]—although this point is much worse documented.
Thus, there is a need to identify more precise ICP and CPP thresholds that account for individual variation in age, sex, disease natural history and treatment intervention. The primary aim of this study is to evaluate, in a non-DC TBI patient population, ICP and CPP thresholds associated with 6-month morbidity and mortality. Further, we wish to evaluate whether these thresholds vary based on the recording period utilised, or based on the day of recording. Finally, secondary aims were to preliminarily assess if these critical thresholds vary based on age and sex.

\section{Methods}

\section{Patient population}

This is an observational cohort study with retrospective analysis of 355 patients within a TBI database. A portion of this population has been described in the previous ICP threshold work done by Sorrentino et al. [22]. These patients were those with a minimum of $6 \mathrm{~h}$ of archived highfrequency physiologic recordings. All patients were admitted to the Neurosciences and Trauma Critical Care Unit (NCCU) at Cambridge University Hospitals NHS Foundation Trust (CUH) between March 2005 and December 2016. Patients suffered either moderate-tosevere TBI or mild TBI and subsequently deteriorated to a point where they required ICP monitoring and sedation and mechanical ventilation as part of ICP management. As such, the timing of ICP monitoring, both in duration and time to initiation after injury, was variable. Treatment received included standard ICP-directed therapy, with an ICP goal of less than $20 \mathrm{mmHg}$ and CPP goal of greater than $60 \mathrm{mmHg}$.

Age, sex, Glasgow Coma Scale (GCS) at admission, injury severity score (ISS) and Glasgow Outcome Scale (GOS) at 6 months were prospectively collected and recorded in the database. Two binary outcome classifications were recorded for each patient at 6 months, namely, alive vs dead and favourable vs unfavourable outcome. Unfavourable outcome was defined as GOS $<4$.

All data were fully anonymised, and no attempt was made to re-access clinical records for additional information. As such, formal patient or proxy consent was not required. Within our institution, patient data may be collected with a waiver of formal consent, as long as it remains fully anonymised, with no method of tracing this back to an individual patient. Patient physiologic, demographic and outcome data were collected by the clinicians involved with patient care and subsequently recorded in an anonymous format. This anonymous data is then provided for future research purposes. Such data curation remains within compliance for research 
integrity as outlined in the Governance Arrangements for Research Ethics Committees (GAfREC), September 2011 guidelines, section $6.0[17,25]$.

The exact number of overlapping patients between the current work and Sorrentino et al. is unknown. As our local prospective signal database is a fluid entity, with patient data added at various points during their hospital stay, we are unable to determine which patients exactly overlap between these two works. In addition, we do not have access to the original anonymous patient identifier codes from the Sorrentino work, further impeding our ability to give exact numbers of overlap.

\section{Signal acquisition and processing}

Intracranial pressure was acquired via an intraparenchymal strain gauge probe (Codman ICP MicroSensor; Codman \& Shurtleff Inc., Raynham, MA). Arterial blood pressure was obtained through either radial or femoral lines connected to pressure transducers (Baxter Healthcare Corp. CardioVascular Group, Irvine, CA). All signals from the above devices were sampled at minimum $50 \mathrm{~Hz}$ and recorded using digital data transfer or digitised via an $A / D$ converter (DT9801 or DT9803; Data Translation, Marlboro, MA), where appropriate, using the ICM+ software (Cambridge Enterprise Ltd., Cambridge, UK, http:// icmplus.neurosurg.cam.ac.uk). Signal artefacts were removed manually prior to further processing or analysis. CPP was determined as the difference between mean arterial pressure and ICP.

Minute-by-minute data processed originally by ICM+ were exported into comma separated values format (CSV). Using $\mathrm{R}$ statistical software ( $\mathrm{R}$ Core Team (2016). R: A language and environment for statistical computing. R Foundation for Statistical Computing, Vienna, Austria. URL https://www.R-project.org/), various data sheets were created including grand mean over entire recording period, first $24 \mathrm{~h}$, first $72 \mathrm{~h}$, first $120 \mathrm{~h}$ and first $168 \mathrm{~h}$. Furthermore, data was also averaged for each day of recording in order to assess time dependence of the critical thresholds.

\section{Statistics}

All statistics were completed using $\mathrm{R}$ statistical software. Descriptive statistics were applied to summarise demographic data. Normality was tested using the ShapiroWilk test for demographic variables and measured indices, all of which were determined to be non-parametric. Non-parametric tests of independence were used to compare demographic and clinical characteristics in patients of each binary outcome classification. Critical thresholds for outcome were derived using sequential chi-square analysis. This method has been previously applied in other publications assessing thresholds for ICP, CPP and continuous autoregulation indices in TBI [21, 22, 27], and its use allowed direct comparison of time-, duration- and treatment-dependent results with existing thresholds. Sequential $2 \times 2$ binary outcome contingency tables were constructed, grouping patients by (1) survival or dichotomised outcome (GOS $\geq 4$ vs GOS $<4$ ) and (2) average ICP and CPP greater or less than sequential thresholds in 0.1 and $0.5 \mathrm{mmHg}$ increments respectively. The ICP or CPP threshold returning the highest chi-square test statistic value was assumed to have the best discriminative value.

The same analysis was repeated for each subgroup of sex (male vs female) and age $(\leq 55$ vs $<55)$. Age categories were set to mirror those used in the initial landmark threshold analysis [22]. Whole cohort and subgroup analysis was repeated for ICP and CPP values averaged over the first $24 \mathrm{~h}$ and 3, 5, 7 and 10 days of ICP monitoring to create duration-of-monitoring-dependent thresholds. Subsequently, the same analysis was repeated again for ICP and CPP means in $24 \mathrm{~h}$ slices to determine critical thresholds for each day of the first 7 days of ICP monitoring. Statistical significance was set at $p<0.05$ for all results. $p$ values were corrected for multiple testing using the Benjamini and Hochberg FDR method, and the results that remain statistically significant were highlighted. Graphical production was completed using the ggplot2 package in $\mathrm{R}$.

Finally, univariable logistic regression analysis was performed for the statistically significant ICP thresholds, assessing the area under the receiver operating curve (AUC) and 95\% confidence intervals (CI) associated with both dichotomised outcomes. This was conducted across all time periods analysed. This was not conducted for CPP, given poor threshold discrimination on chisquare testing.

\section{Results}

\section{Cohort characteristics}

A total of 355 patients (271 male, 84 female) were included in the study, with a mean age of 40.6 (SD 17.2) and a median admission GCS of 7 (IQR 3-9). The mean duration of ICP monitoring was 6.81 days (SD 5.99). Six months after admission, 172 patients had favourable outcomes as assessed by the Glasgow Outcome Score (low or moderate disability), and 183 had unfavourable outcomes (severe disability, persistent vegetative state or death) of whom 63 had died. Mean age and mean ICP were significantly higher among 
patients who died or had an unfavourable outcome. Mean ICP monitoring period was also higher in patients with unfavourable outcome. Median admission GCS was lower in patients who died or unfavourable outcome, while sex distribution, median ISS and mean CPP were not significantly different (Table 1). Mean ICP and CPP by patient outcome (alive/dead and favourable/ unfavourable) for each day and duration of monitoring are given in Appendix A.

\section{Overall thresholds}

In the whole cohort across the full duration of ICP monitoring, the overall ICP threshold for mortality was $21.3 \mathrm{mmHg}\left(\chi^{2}=\right.$ $42.90, p<0.001)$. The threshold for the unfavourable outcome was $20.5 \mathrm{mmHg}\left(\chi^{2}=20.73, p<0.001\right)$ (Fig. 1).

The ICP thresholds for mortality in patients aged $>55(n=$ $78)$ and $\leq 55(n=277)$ were $14.7 \mathrm{mmHg}\left(\chi^{2}=8.3 ; p=\right.$ $0.0039)$ and $20.7 \mathrm{mmHg}\left(\chi^{2}=39.34 ; p<0.001\right)$ respectively, while the thresholds for unfavourable outcome were $19.9 \mathrm{mmHg}\left(\chi^{2}=4.97 ; p=0.026\right)$ and $20.6 \mathrm{mmHg}\left(\chi^{2}=\right.$ $17.35 ; p<0.001)$ respectively. Male and female patients were also evaluated, but given the small number of female relative to male patients, the strength of conclusions is limited. Sexspecific results are presented in Appendix B. Univariable logistic regression analysis for the statistically significant thresholds can be found in Appendix C, providing AUC and 95\% CI for the dichotomised outcomes across all statistically significant ICP thresholds identified.

\section{Duration-dependent thresholds}

Sequential chi-square analysis was repeated across all subgroups during the first $24 \mathrm{~h}$ and 3, 5 and 7 days of ICP monitoring. The whole cohort results are presented in Fig. 2. Over the first $24 \mathrm{~h}$, the threshold for mortality was higher than the overall threshold $\left(24.7 \mathrm{mmHg} ; \chi^{2}=31.30 ; p<0.001\right)$ then dropped below $20 \mathrm{mmHg}$ over longer periods of monitoring. The threshold for unfavourable outcome remained comparatively stable across all recorded lengths of monitoring, though significant unfavourable outcome thresholds could not be identified at 7 .

The duration-dependent ICP thresholds for males and patients of age $\leq 55$ closely followed the pattern of the overall cohort. The 24-h threshold for the unfavourable outcome was lower in female patients. Female mortality thresholds mirrored overall thresholds over $24 \mathrm{~h}$ and 3 days, but significant thresholds could not be identified beyond this (Appendix B). In older patients, lower thresholds were identified for both mortality and unfavourable outcome in the first $24 \mathrm{~h}$, but further durationdependent thresholds are not significant (Appendix D). It must be acknowledged that the number of patients included dropped over longer durations of recording.

\section{Time-dependent thresholds}

Sequential chi-square analysis was repeated for each 24-h period for the first 7 days of ICP monitoring. In the whole cohort, ICP threshold for mortality dropped from $24.7 \mathrm{mmHg}\left(\chi^{2}=\right.$ $31.30 ; p<0.001)$ in the first day to $16.5 \mathrm{mmHg}\left(\chi^{2}=29.29\right.$; $p<0.001$ ) in the second day and remained below $20 \mathrm{mmHg}$ for each $24 \mathrm{~h}$ period assessed. The threshold for unfavourable outcome dropped from $20.3 \mathrm{mmHg}\left(\chi^{2}=36.41 ; p<0.001\right)$ on the first day to remain at or below $20 \mathrm{mmHg}$ for each day where a significant threshold was identified (Fig. 3).

Time-dependent thresholds in the male and young subgroups were similar to overall cohort. Where significant thresholds were identified for the female subgroup, they were also similar with the exception of a lower day 1 outcome

Table 1 Cohort characteristics

\begin{tabular}{|c|c|c|c|c|c|c|c|}
\hline & Whole cohort & $\begin{array}{l}\text { Favourable } \\
\text { outcome } \\
(\text { GOS } \geq 4)\end{array}$ & $\begin{array}{l}\text { Unfavourable } \\
\text { outcome } \\
(\mathrm{GOS}<4)\end{array}$ & $p$ value & Survived & Died & $p$ value \\
\hline Number of patients & 355 & 172 & 183 & & 292 & 63 & \\
\hline Sex & & & & 0.76 & & & 0.27 \\
\hline Male & 271 & 133 & 138 & & 219 & 52 & \\
\hline Female & 84 & 39 & 45 & & 73 & 11 & \\
\hline Mean age (SD) & $40.6(17.2)$ & $38.2(17.0)$ & $43.0(17.0)$ & 0.0091 & $39.4(16.6)$ & $46.6(18.5)$ & 0.0026 \\
\hline Mean ICP (mmHg) (SD) & $14.1(7.7)$ & $12.6(4.6)$ & $15.4(19.5)$ & 0.0039 & $12.9(5.8)$ & $19.4(12.0)$ & $<0.001$ \\
\hline Mean CPP (mmHg) (SD) & $77.5(8.5)$ & $77.6(6.7)$ & $77.6(9.9)$ & 0.31 & $77.9(7.5)$ & $76.2(11.9)$ & 0.39 \\
\hline Mean ICP monitoring period (days) (SD) & $6.81(5.99)$ & $6.39(5.50)$ & $7.21(6.38)$ & 0.0030 & $6.95(6.21)$ & $6.21(4.78)$ & 0.31 \\
\hline Median admission GCS (IQR) & $7(3.25-9)$ & $8(5-11)$ & $6(3-8)$ & $<0.001$ & $7(4-10)$ & $4(3-8)$ & 0.0026 \\
\hline Median ISS & $33(25-41)$ & $31.5(25-38)$ & $33(25-41)$ & 0.20 & 33 & 29.5 & 0.76 \\
\hline
\end{tabular}

$C P P$ cerebral perfusion pressure, GCS Glasgow Coma Scale, GOS Glasgow Outcome Score, ICP intracranial pressure, IQR interquartile range, ISS injury severity score, $S D$ standard deviation 


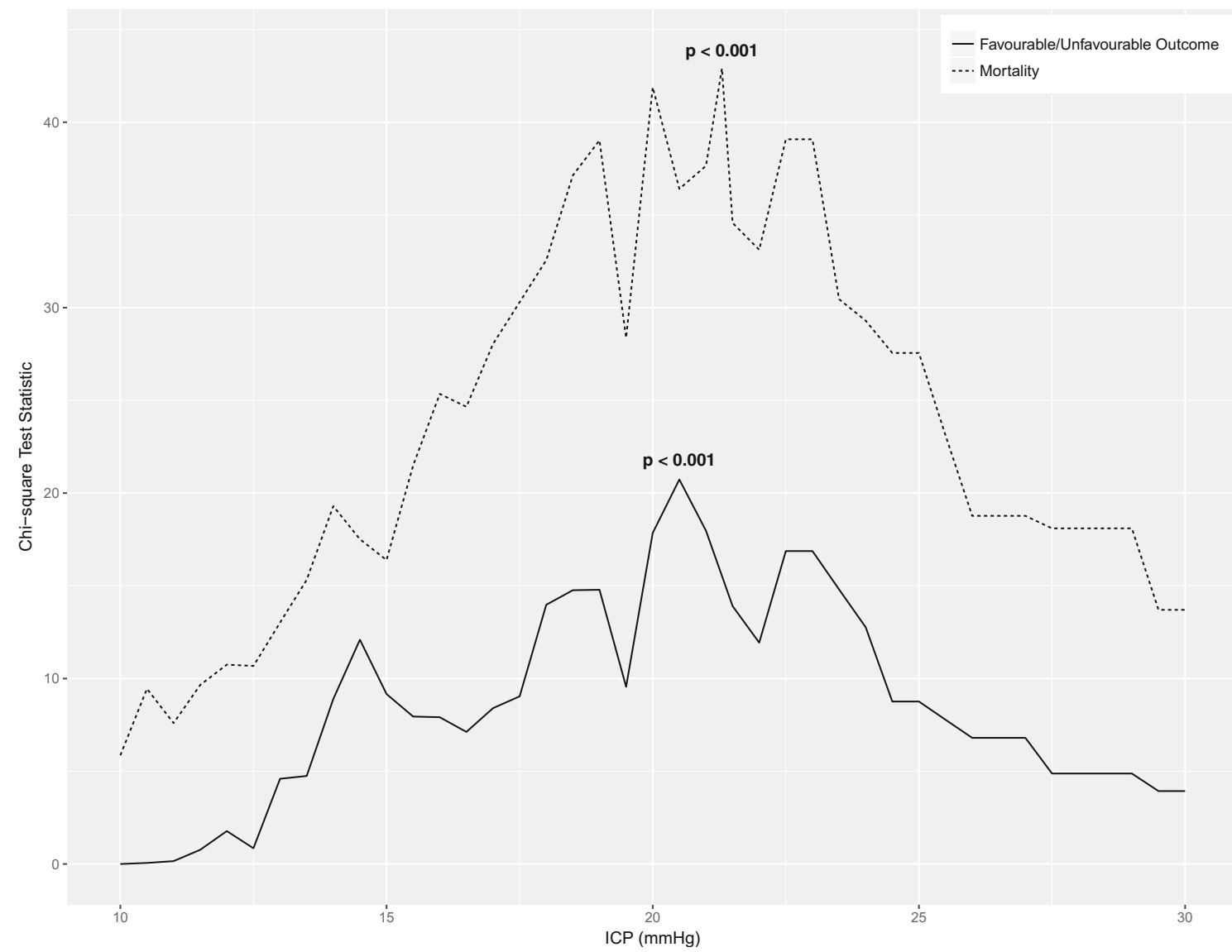

Fig. 1 Sequential chi-square analysis for intracranial pressure threshold. The values returning the highest chi-square test statistic, and therefore having the highest discriminatory value for outcome, were taken as

threshold (Appendix B). Patients aged $>55$ had lower ICP thresholds for both mortality and unfavourable outcome for the first 2 days of monitoring, but significant thresholds were not identified for later days (Appendix D).

\section{Discussion}

\section{ICP}

This study presents the first systematic analysis of timedependent ICP thresholds for mortality and unfavourable outcome in TBI patients managed without DC. The overall thresholds, based on the entire recording period, that best discriminate mortality and unfavourable outcome were 21.3 and $20.5 \mathrm{mmHg}$ respectively. These thresholds were the most statistically significant ICP thresholds associated with global patient outcome, more so than a threshold of 20 (i.e. a commonly applied treatment threshold in clinical practice). They indicate that patients with mean ICP values above these particular thresholds had the strongest association with worse dichotomised global outcomes at 6 months. These are slightly below the universal threshold of $22 \mathrm{mmHg}$ recommended in thresholds and are denoted by $p$ values. Bold $p$ values remained significant on correction for multiple comparisons. ICP denotes intracranial pressure

the current consensus guidelines [8], though they remain in the $20-25 \mathrm{mmHg}$ range usually applied in clinical practice. However, time-specific analysis revealed variable ICP thresholds over the first week of monitoring. After the first $24 \mathrm{~h}$ of monitoring, the daily thresholds fell and remained between 15 and $20 \mathrm{mmHg}$. This was apparent both when the data was grouped by duration of monitoring and analysed on a dayby-day basis.

Sex-specific analysis revealed female patients had a higher overall ICP threshold for mortality, but the duration- and timedependent ICP thresholds followed a similar pattern to those of male patients, with only occasional variations. Given the low number of female patients in the cohort, it is not possible to comment definitively on significant sex differences in ICP threshold based on this data alone. It is interesting to note lower ICP thresholds for mortality and unfavourable outcome in older patients, both overall and in the first 2 days of ICP monitoring. Though carrying a similar caveat due to the low proportion of older patients, this is in keeping with previous evidence that older patients may exhibit higher vulnerability to ICP insult [22], particularly in the early stages of ICP management. It is also notable that chi-square test statistic values are generally substantially higher in mortality thresholds for 


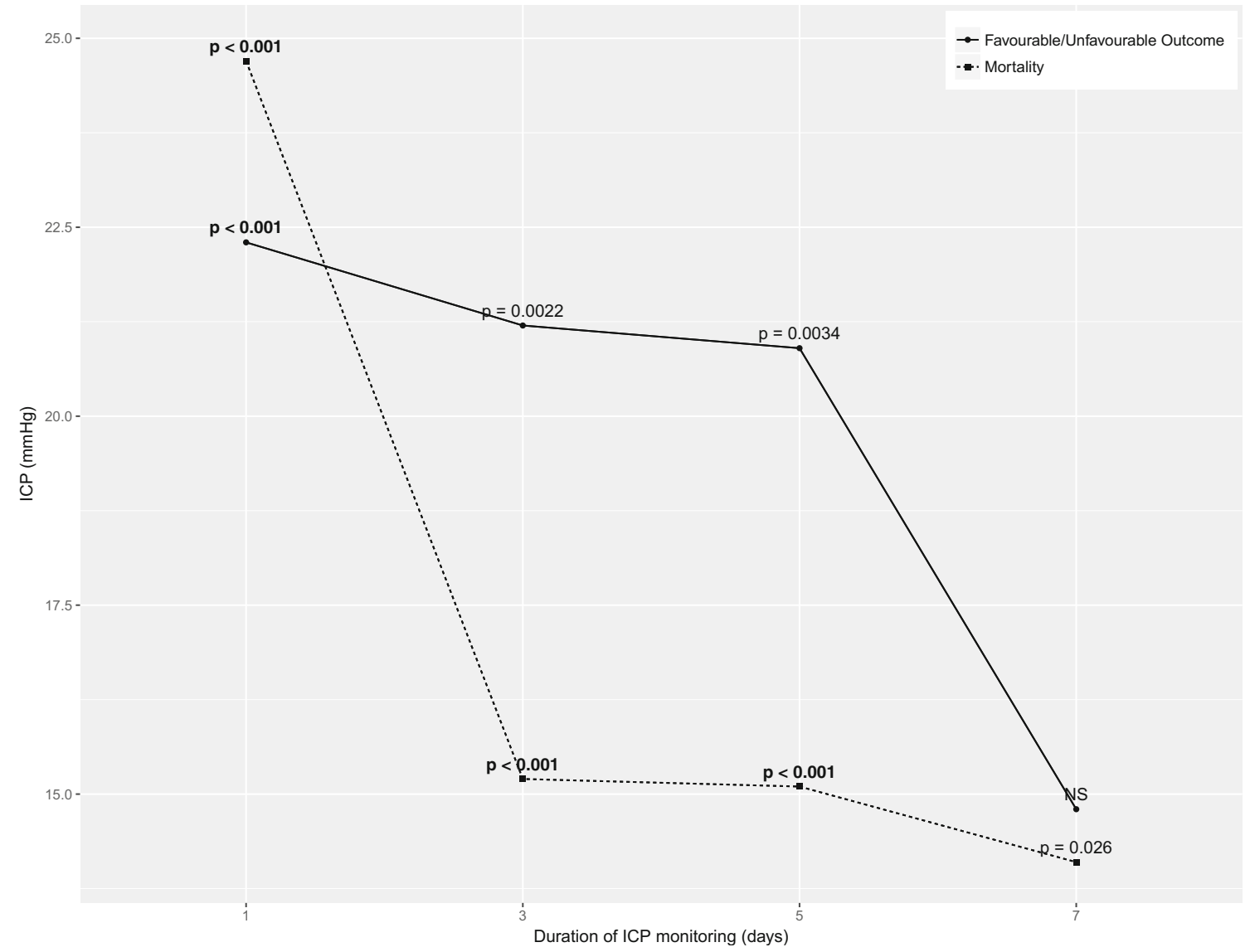

Fig. 2 Whole cohort thresholds by duration of monitoring ICP. Bold $p$ values remained significant on correction for multiple comparisons. Number of patients with available ICP data over each monitoring

both ICP and CPP, suggesting a stronger association between these metrics and mortality as compared with the unfavourable outcome. This is concordant with previous results [22].

Our results establish ICP thresholds for non-DC patients that are slightly distinct from the threshold previously described for a mixed cohort [22]. Moreover, it is unsurprising to find these thresholds differ to those recently described for DC patients [19]. These results, taken together, reinforce the need to develop individualised ICP thresholds, as treatment modalities and patient characteristics introduce tremendous heterogeneity.

The ultimate goal is to derive ICP thresholds that derive rationally from patient-specific pathophysiology. There is evidence that the tolerability of ICP insults vary between patients depending on autoregulatory capacity [13] and intracranial pressure-volume dynamics [7]. Lazardis et al. [14] used a pressure reactivity index $(\mathrm{PRx}$ - correlation between ICP and MAP) cut-off of $>0.2$ to define patient-specific ICP thresholds at which cerebrovascular pressure reactivity was deranged. These variable thresholds yielded superior outcome prediction than fixed thresholds of 20 or $25 \mathrm{mmHg}$. In the period were as follows: $24 \mathrm{~h}, 340 ; 3$ days, 277; 5 days, 217; and 7 days, 163. ICP denotes intracranial pressure; NS not significant

context of our findings, which show a time-dependent ICP threshold that usually remains below $20 \mathrm{mmHg}$, pulse amplitude index (PAx - correlation between pulse amplitude of ICP and MAP) may be superior for the latter strategy, as it is proven to be a superior outcome predictor than PRx at ICP $<20 \mathrm{mmHg}$ [4].

\section{CPP}

We explored CPP thresholds using the same methodology. The results showed no identifiable trends, and significant time-dependent thresholds for mortality or unfavourable outcome were often not identified, even in the whole cohort and in well-populated subgroups (males and young patients). As no significant conclusions could be drawn from these results, they have not been included in the manuscript.

This lack of ability to determine global clinically significant CPP thresholds likely stems from the issue of targeting a global CPP target for everyone. CPP thresholds in TBI have remained relatively elusive for some time now, with conflicting literature on appropriate CPP ranges to target, leading to changes in recommendations between various renditions of 


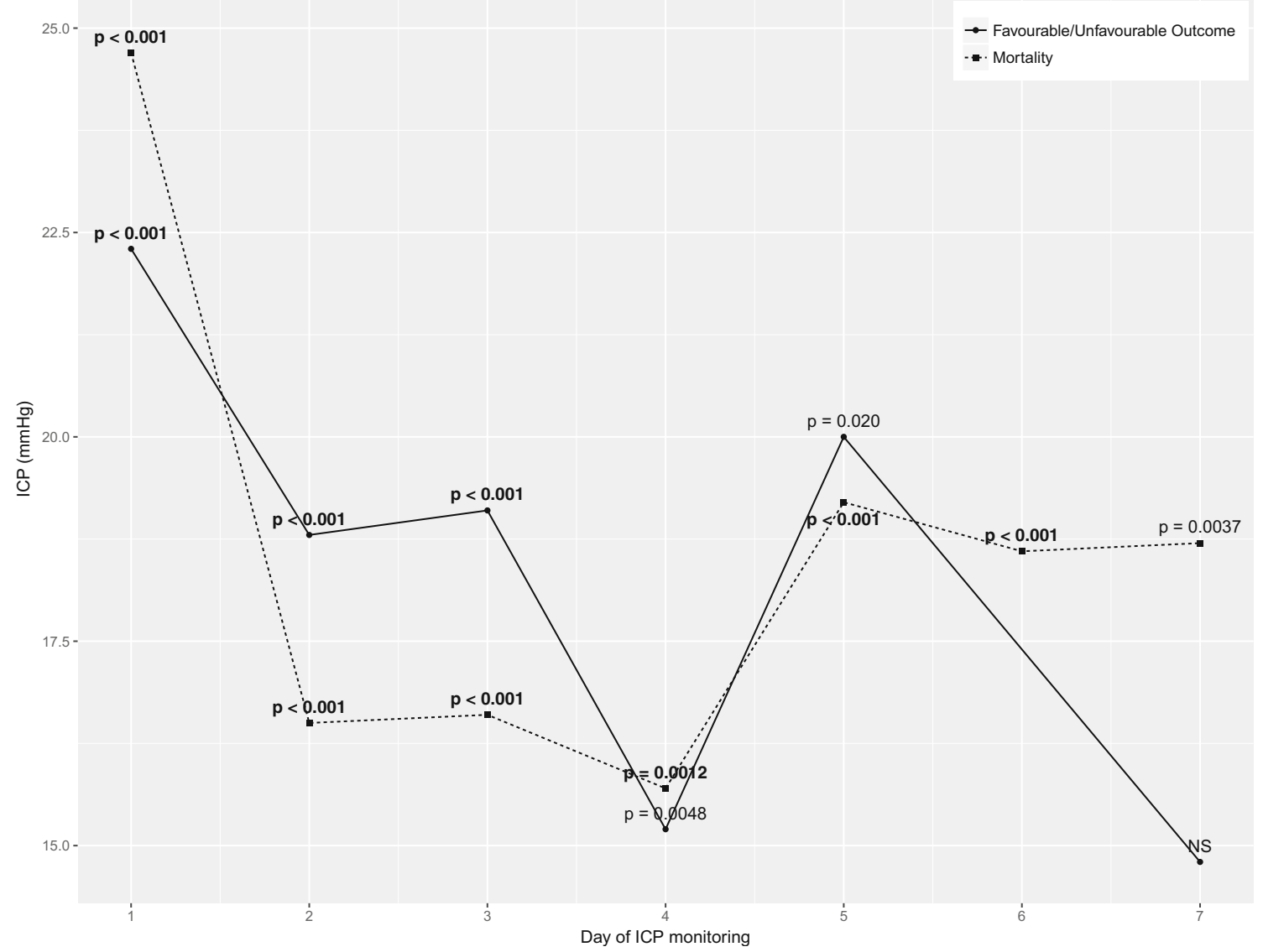

Fig. 3 Whole cohort ICP thresholds by day of monitoring. Not significant ICP outcome point at 6 days removed. Bold $p$ values remained significant on correction for multiple comparisons. Number of patients with available ICP data for each day of monitoring were as

the BTF guidelines $[6,8]$. Though the lack of significant results within this study does not provide definitive evidence, we believe they do provide further support of 'personalised' CPP targets in TBI. Such personalised CPP targets include those suggested by the CPP optimum (CPPopt) concept, with numerous papers to date suggesting a stronger link between CPPopt and patient outcome [18]. The definitive link between these personalised CPP targets in TBI has yet to be determined, with prospective randomised trials underway [COGITATE, ClinicalTrials.gov Identifier: NCT02982122].

We do not believe that the lack of results for CPP suggests that ICP is more important and should be preferentially targeted in TBI therapy. It is likely the way we currently view CPP is the issue, arguing for a change in the way we analyse and target it.

\section{Limitations}

First, this study is limited by its retrospective design, making it impossible to control for the influence of treatment targets on the derived thresholds. Additionally, as high ICP may reflect the global severity of a patient's condition, it is impossible to determine the direct effect of ICP thresholds on the outcome follows: day 1,340 ; day 2,296 ; day 3,270 ; day 4,234 ; day 5,199 ; day 6,176; and day 7,144. ICP denotes intracranial pressure; NS not significant

without prospective validation. Furthermore, as these data were collected over 11 years, changing management patterns over time may confound the results. Given the potential for treatment heterogeneity across our patient population, this needs further acknowledgement as a limitation. The information within our database was retrospectively accessed for the purpose of this study, without the ability to re-access charts for missing information. Thus, comments on treatment intensity (i.e. doses of hypertonic agents, use of sedation, cerebrospinal fluid drainage, use of barbiturates) and its potential relation to the thresholds seen cannot be made based on this data. This is an important limitation of our study, as not only can various treatments directly impact the ICP and CPP values recorded, but also those patients with medically refractory ICP may have had a reduction on therapy secondary to futility. Such a reduction in therapy could lead to persistent ICP elevations skewing the thresholds found within our study. However, with that said, we believe that the number of patients that would fall into this category would be quite small within the population studied, as those with persistent refractory ICP issues typically undergo a secondary DC at our institution. All DC patients were excluded from this study to avoid such confounding. 
Thus, despite the absence of information on treatment intensity, we are confident that the results presented are not significantly skewed due to outliers.

Furthermore, despite the exclusion of DC patients from our study, there were subjects in our study population that had medically refractory ICP (i.e. levels persistently above $20 \mathrm{mmHg}$ during a large portion of their ICP stay). It is unknown why these patients did not undergo DC, as this was not recorded within our database that was retrospectively accessed for the purpose of this study. It is possible that these patients represent those that were 'too sick' for surgical salvage therapy. This further adds to the heterogeneity of our TBI population for this study, thus re-emphasising that the results found for ICP and CPP thresholds are preliminary, with further validation in a multicentre cohort required.

Second, although 355 patients were included in the study, the characteristic demographic pattern of TBI meant that the statistical power of less well-populated subgroup analysis was weakened by small sample size, notably in females and patients aged $>55$. This may explain the relative absence of statistically significant thresholds in these subgroups especially. Thus, we were unable to definitively comment on whether there is a significant difference in ICP thresholds between age and sex groups. There exists the potential that age-related cerebral atrophy can confound the ICP thresholds seen. With advanced age, cerebral atrophy increases. Thus, to reach the same ICP values as younger patients, one could argue that more severe intracranial injury is required in these elderly patients, with larger mass lesions/oedema volume required. Thus, when assessing ICP thresholds associated with outcome, this potential injury severity discrepancy between the young and elderly, with similar ICP values, may impact the results. Based on the data available in our database, we were unable to assess this. We are planning a follow-up study with the ICU cohort from CENTER-TBI [15], using upwards of 2000 patients, with detailed imaging assessments, to probe into age-specific ICP thresholds and the impact of intracranial injury patterns.

Third, the time-dependent analysis is limited by values of ICP averaged over $24 \mathrm{~h}$. Therefore, it was not possible in this work to identify time-dependent critical thresholds over a shorter timescale. Moreover, the average ICP values do not directly relate to time spent above putative thresholds, or ICP 'dose' $[13,26]$. The drop in ICP-based critical thresholds after the first $24 \mathrm{~h}$ of recording may be secondary to either treatment effect, or reflect the 'dose' response to ICP, where absolute thresholds may be less useful than the amount of time spent above such values $[13,26]$. While this generally did not have an effect on the derived ICP thresholds, the accuracy of the sequential chi-square method of threshold analysis is limited at extreme ICP values by small cell sizes.

Fourth, a further limitation of this retrospective database study is lack of clarity on cause of mortality. The information available within our dataset includes only a rough global assessment of patient outcome at 6-months (i.e. GOS), without elaboration on cause of mortality. Thus, we are unable to separate those who died secondary to neurological causes from other in-hospital causes within this study. This would have provided potentially interesting insight into differences in ICP and CPP thresholds based on mortality causes. Given this limitation within our study, this will be assessed in the upcoming analysis of the multicentre high-resolution ICU cohort in the CENTER-TBI [15], with the hope that further light may be shed on this topic.

Fifth, DC patients were specifically excluded to avoid the potential for confounding effects on the ICP thresholding. Much additional work is required in this population to assessed ICP and CPP critical thresholds associated with global outcome. In the presence of DC, the value returned from ICP monitoring is exceedingly difficult to interpret, given an open cranial vault and influence from atmospheric pressure on the readings. Thus, despite the difference in ICP threshold discovered between our work and the original Sorrentino study, we refrain from making generalised comments on ICP thresholds from DC patient populations at this time, until further objective analysis of this population is conducted. This work is currently under way.

Finally, it is important to emphasise that this work is preliminary. Thus, although we have identified differing trends within the non-DC population, and a change in threshold over time, further confirmatory work is required prior to any change in guideline-based threshold targets. We must re-emphasise that the results of this analysis should not change current ICP treatment thresholds recommended in current guidelines. In addition, having separate thresholds for mortality and favourable/unfavourable outcome is not entirely clinically useful, as it pertains to threshold targeting for ICP-directed therapies. These different thresholds only provide information regarding potentially predicting functional outcome. We plan to undertake further prospective analysis using the ICU cohort data-set from CENTER-TBI, to better define ICP thresholds over time and perform more detailed subgroup analysis.

\section{Conclusion}

A global ICP threshold for non-DC TBI patients is identified as $21.3 \mathrm{mmHg}$ for mortality and $20.5 \mathrm{mmHg}$ for unfavourable outcome, similar to what has been described in the BTF guidelines. However, after the first day of ICP monitoring, timedependent ICP thresholds fell to between 15 and $20 \mathrm{mmHg}$, lower than the targets recommended in current guidelines and used in clinical practice. These thresholds are lower still in older patients. These results underscore the importance of developing individualised ICP thresholds based on demographics, treatment modalities, patient-specific pathophysiology and, possibly, other physiological markers. 
Acknowledgements This work was made possible through salary support through the Cambridge Commonwealth Trust Scholarship, the Royal College of Surgeons of Canada-Harry S. Morton Travelling Fellowship in Surgery and the University of Manitoba Clinician Investigator Program.

These studies were supported by the National Institute for Healthcare Research (NIHR, UK) through the Acute Brain Injury and Repair theme of the Cambridge NIHR Biomedical Research Centre, an NIHR Senior Investigator Award to DKM. The authors were also supported by a European Union Framework Program 7 grant (CENTER-TBI; Grant Agreement No. 602150).

MC is supported by a grant of the Korea Health Technology R\&D Project through the Korea Health Industry Development Institute (KHIDI) funded by the Ministry of Health and Welfare, Republic of Korea (grant number: HI17C1790)

\section{Compliance with ethical standards}

Disclosures FAZ has received salary support for dedicated research time, during which this project was partially completed. Such salary support came from the Cambridge Commonwealth Trust Scholarship, the Royal College of Surgeons of Canada-Harry S. Morton Travelling Fellowship in Surgery and the University of Manitoba Clinician Investigator Program.

DKM has consultancy agreements and/or research collaborations with GlaxoSmithKline Ltd.; Ornim Medical; Shire Medical Ltd.; Calico Inc.; Pfizer Ltd.; Pressura Ltd.; Glide Pharma Ltd.; and NeuroTraumaSciences LLC.

MC and PS have financial interest in a part of licencing fee for ICM+ software (Cambridge Enterprise Ltd., UK).

Ethical approval All procedures performed in studies involving human participants were in accordance with the ethical standards of the institutional and/or national research committee and with the 1964 Helsinki declaration and its later amendments or comparable ethical standards. For this type of study, formal consent is not required.

Informed consent All data was fully anonymised, and no attempt was made to re-access clinical records for additional information. As such, formal patient or proxy consent was not required. Within our institution, patient data may be collected without consent, as long as it remains fully anonymised, with no method of tracing this back to an individual patient. Such requests for data curation in our TBI population have gone through our local Research Committee, as part of an NCCU protocol (Protocol \#30), achieving approval for ongoing collection of such data, without the need for formal patient or proxy consent.

Open Access This article is distributed under the terms of the Creative Commons Attribution 4.0 International License (http:// creativecommons.org/licenses/by/4.0/), which permits unrestricted use, distribution, and reproduction in any medium, provided you give appropriate credit to the original author(s) and the source, provide a link to the Creative Commons license, and indicate if changes were made.

\section{References}

1. Alali AS, Fowler RA, Mainprize TG et al (2013) Intracranial pressure monitoring in severe traumatic brain injury: results from the American College of Surgeons Trauma Quality Improvement Program. J Neurotrauma 30(20):1737-1746

2. Allen BB, Chiu YL, Gerber LM, Ghajar J, Greenfield JP (2014) Age-specific cerebral perfusion pressure thresholds and survival in children and adolescents with severe traumatic brain injury. Pediatr Crit Care Med 15(1):62

3. Aries MJ, Czosnyka M, Budohoski KP et al (2012) Continuous determination of optimal cerebral perfusion pressure in traumatic brain injury. Crit Care Med 40(8):2456-2463

4. Aries MJ, Czosnyka M, Budohoski KP et al (2012) Continuous monitoring of cerebrovascular reactivity using pulse waveform of intracranial pressure. Neurocrit Care 17(1):67-76

5. Balestreri M, Czosnyka M, Hutchinson $P$ et al (2006) Impact of intracranial pressure and cerebral perfusion pressure on severe disability and mortality after head injury. Neurocrit Care 4(1):8-13

6. Bratton SL, Chestnut RM, Ghajar J et al (2007) Guidelines for the management of severe traumatic brain injury. IX. Cerebral perfusion thresholds. J Neurotrauma 24:S59-S64

7. Calviello L, Donnelly J, Cardim D et al (2017) Compensatoryreserve-weighted intracranial pressure and its association with outcome after traumatic brain injury. Neurocrit Care:1-9

8. Carney N, Totten AM, O'reilly C et al (2017) Guidelines for the management of severe traumatic brain injury. Neurosurgery 80(1): $6-15$

9. Chesnut RM, Temkin N, Carney N et al (2012) A trial of intracranial-pressure monitoring in traumatic brain injury. $\mathrm{N}$ Engl J Med 367(26):2471-2481

10. Eisenberg HM, Frankowski RF, Contant CF et al (1988) High-dose barbiturate control of elevated intracranial pressure in patients with severe head injury. J Neurosurg 69(1):15-23

11. Farahvar A, Gerber LM, Chiu YL et al (2012) Increased mortality in patients with severe traumatic brain injury treated without intracranial pressure monitoring. J Neurosurg 117(4):729-734

12. Gerber LM, Chiu YL, Carney N, Härtl R, Ghajar J (2013) Marked reduction in mortality in patients with severe traumatic brain injury. J Neurosurg 119(6): 1583-1590

13. Güiza F, Depreitere B, Piper I et al (2015) Visualizing the pressure and time burden of intracranial hypertension in adult and paediatric traumatic brain injury. Intensive Care Med 41(6):1067-1076

14. Lazaridis C, DeSantis SM, Smielewski P et al (2014) Patientspecific thresholds of intracranial pressure in severe traumatic brain injury. J Neurosurg 120(4):893-900

15. Maas AI, Menon DK, Steyerberg EW et al (2014) Collaborative European NeuroTrauma Effectiveness Research in Traumatic Brain Injury (CENTER-TBI): a prospective longitudinal observational study. Neurosurgery 76(1):67-80

16. Marmarou A, Anderson RL, Ward JD et al (1991) Impact of ICP instability and hypotension on outcome in patients with severe head trauma. Spec Suppl 75(1S):S59-S66

17. National Research Ethics Service (2012) "Changes to the remit of Research Ethics Committees (September 2011)" https://www. research-integrity.admin.cam.ac.uk/files/gafrec_-_changes_to the recs v1.1 march 20121.pdf. Accessed 7 April 2018

18. Needham E, McFadyen C, Newcombe V et al (2017) Cerebral perfusion pressure targets individualized to pressure-reactivity index in moderate to severe traumatic brain injury: a systematic review. J Neurotrauma 34(5):963-970

19. Sauvigny T, Göttsche J, Czorlich P, Vettorazzi E, Westphal M, Regelsberger J (2017) Intracranial pressure in patients undergoing decompressive craniectomy: new perspective on thresholds. J Neurosurg:1-9

20. Schreiber MA, Aoki N, Scott BG, Beck JR (2002) Determinants of mortality in patients with severe blunt head injury. Arch Surg 137(3):285-290

21. Sorrentino E, Budohoski KP, Kasprowicz M et al (2011) Critical thresholds for transcranial Doppler indices of cerebral autoregulation in traumatic brain injury. Neurocrit Care 14(2):188-193

22. Sorrentino E, Diedler J, Kasprowicz M et al (2012) Critical thresholds for cerebrovascular reactivity after traumatic brain injury. Neurocrit Care 16(2):258-266 
23. Steiner LA, Czosnyka M, Piechnik SK et al (2002) Continuous monitoring of cerebrovascular pressure reactivity allows determination of optimal cerebral perfusion pressure in patients with traumatic brain injury. Crit Care Med 30(4):733-738

24. Talving P, Karamanos E, Teixeira PG et al (2013) Intracranial pressure monitoring in severe head injury: compliance with Brain Trauma Foundation guidelines and effect on outcomes: a prospective study. J Neurosurg 119(5):1248-1254

25. UK Health Departments (2011) "Governance arrangements for research ethics committees: a harmonised edition" https://assets.publishing.service.gov.uk/government/uploads/ system/uploads/attachment_data/file/213753/dh_133993.pdf. Accessed 7 April 2018

26. Vik A, Nag T, Fredriksli OA, et al (2008). Relationship of "dose" of intracranial hypertension to outcome in severe traumatic brain injury

27. Zeiler FA, Cardim D, Donnelly J et al (2018) Transcranial Doppler systolic flow index and ICP-derived cerebrovascular reactivity indices in traumatic brain injury. J Neurotrauma 35(2):314-322 